\title{
The surgical management of early-stage lung adenocarcinoma: is wedge resection effective?
}

\author{
Ying Ji ${ }^{1 \#}$, Guangyu Bai ${ }^{1 \#}$, Bin Qiu ${ }^{1 \#}$, Liang Zhao ${ }^{1}$, Jing Zhou ${ }^{2}$, Qi Xue ${ }^{1}$, Shugeng Gao ${ }^{1}$ \\ ${ }^{1}$ Department of Thoracic Surgery, National Cancer Center/National Clinical Research Center for Cancer/Cancer Hospital, Chinese Academy of \\ Medical Sciences and Peking Union Medical College, Beijing, China; ${ }^{2}$ School of Statistics, Renmin University of China, Beijing, China \\ Contributions: (I) Conception and design: Y Ji, G Bai; (II) Administrative support: S Gao, B Qiu; (III) Provision of study materials or patients: B Qiu, \\ L Zhao, Q Xue, S Gao; (IV) Collection and assembly of data: Y Ji, G Bai; (V) Data analysis and interpretation: J Zhou, Y Ji, G Bai; (VI) Manuscript \\ writing: All authors; (VII) Final approval of manuscript: All authors. \\ "These authors contributed equally to this work. \\ Correspondence to: Shugeng Gao, MD. Department of Thoracic Surgery, National Cancer Center/National Clinical Research Center for Cancer/Cancer \\ Hospital, Chinese Academy of Medical Sciences and Peking Union Medical College, Beijing 100021, China. Email: gaoshugeng@cicams.ac.cn.
}

\begin{abstract}
Background: The aim of the study was to explore the outcomes of wedge resection on patients with earlystage lung adenocarcinoma (LUAD) and further identify potential prognostic factors for these patients.

Methods: A retrospective cohort of 190 patients (99 solitary LUAD and 91 multifocal LUAD) undergone wedge resection from October 2014 to September 2015 was established. Cox proportional-hazards model was used to evaluate the significant clinical prognostic factors. Further, data on patients with multifocal adenocarcinoma after segmentectomy were retrieved and propensity score matching was used to compare the outcomes of patients with multiple pulmonary nodules (MPNs) after wedge resection and segmentectomy.

Results: The 5-year overall survival (OS), progression-free survival (PFS), and lung cancer specific survival of the 190 patients after wedge resection were $95.5 \%, 87.9 \%$, and $97.7 \%$, respectively. Multivariable analysis showed that MPN [hazard ratio (HR) 3.07; 95\% confidence interval (CI), 1.05-8.98] and solid-dominant lesions (HR 15.87; 95\% CI, 2.38-105.84) were independently associated with worse PFS. Further, propensity score matching analysis showed that MPN patients had better PFS after segmentectomy compared with wedge resection ( $94 \%$ vs. $80.9 \%, \mathrm{P}=0.008$ ). MPN patients were more likely to perform systematic mediastinal nodal sampling $(95.6 \%$ vs. $59.3 \%, \mathrm{P}<0.001)$ after segmentectomy compared to patients who underwent wedge resection.

Conclusions: Wedge resection is a practical option for appropriately selected early-stage LUAD where tumor size is less than $2 \mathrm{~cm}$ and has a consolidation-to-tumor ratio $\leq 0.5$. However, for MPNs, wedge resection may be not reliable and alternative procedures such as segmentectomy should be used.
\end{abstract}

Keywords: Wedge resection; segmentectomy; early-stage lung cancer; surgical oncology

Submitted Sep 29, 2020. Accepted for publication Feb 06, 2021.

doi: $10.21037 /$ jtd-20-3005

View this article at: http://dx.doi.org/10.21037/jtd-20-3005

\section{Introduction}

Lung cancer is the leading cause of cancer-related death worldwide, with non-small cell lung cancer (NSCLC) accounting for about $85 \%$ of the incident cases, causing a five-year survival rate of $19 \%(1,2)$. At present, an increasing number of early-stage lung cancer can be detected using thin-section computed tomography (TSCT).
Although, the current gold standard surgical procedure for NSCLC is still lobectomy $(3,4)$, sublobar resection (SLR) which includes wedge resection and segmentectomy have been reported to be effective by GGOs (ground-glass opacities) (5-8). Further, SLR is recommended for earlystage NSCLC patients with low tolerance for lobectomy (9-11). 


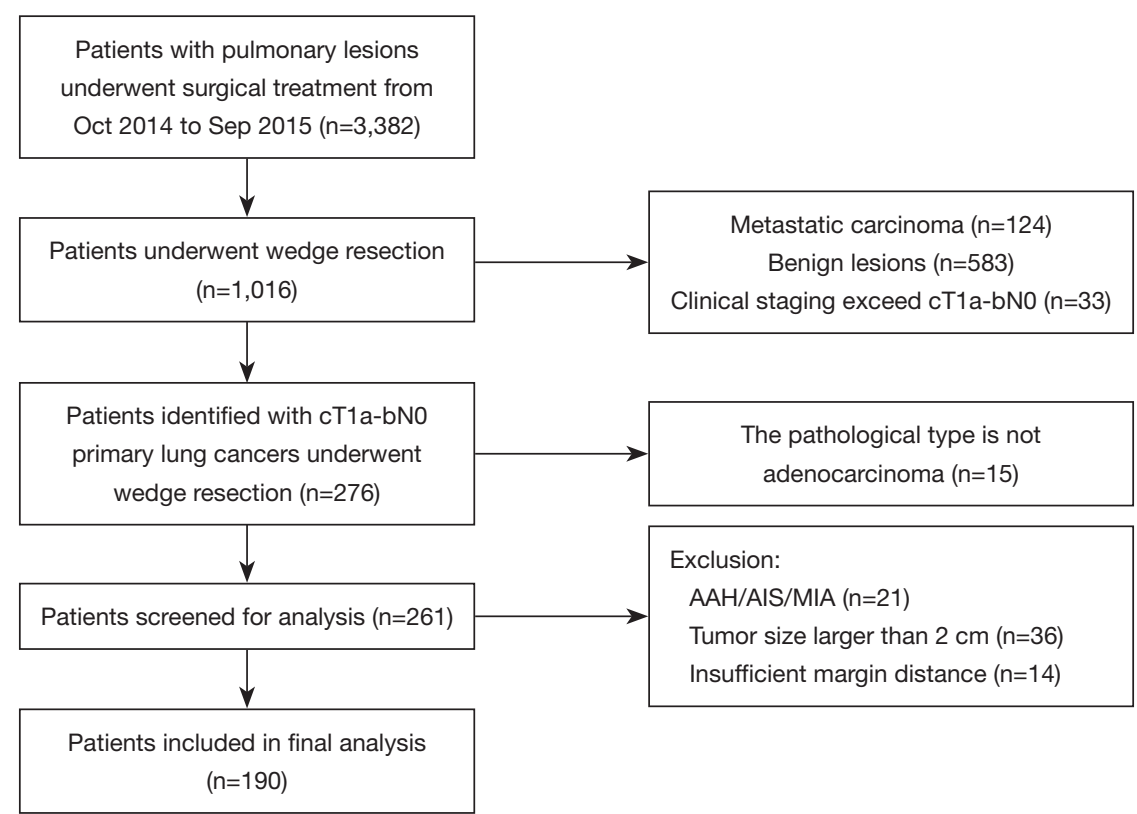

Figure 1 The flow diagram of the patient selection. AAH, atypical adenomatous hyperplasia; AIS, adenocarcinoma in situ; MIA, microinvasive adenocarcinoma.

Although, several studies have compared survival outcome of wedge resection and segmentectomy $(12-16)$, it is not clear which procedure is more reliable for peripheral lung nodules. Most studies have reported similar prognosis for segmentectomy and wedge resection on patients with stage IA NSCLC less than $1.0 \mathrm{~cm}(17,18)$, but the outcomes were unclear for 1.0-2.0 cm tumors with different consolidation-to-tumor ratio (CTR) (19-21). Besides, an international consensus has not been reached for an effective surgery procedure for synchronous multifocal nodules proven or suspected to be multiple primary lung cancer. Although a meta-analysis published in 2019 recommended that SLR is effective for multifocal nodules (22), the wedge resection outcomes of patients with multiple pulmonary nodules (MPNs) remain unclear and few studies have reported comparison of MPN prognosis and solitary pulmonary nodule (SPN).

It is widely accepted that "margin distance (MD)" is a crucial factor affecting the prognosis of wedge resection (23-25). In this study, we aimed at exploring the outcomes of wedge resection with sufficient $M D$ (MD $\geq 20 \mathrm{~mm}$ or larger than tumor size) and identify prognostic factors of patients with early lung adenocarcinoma (LUAD) after undergoing wedge resection. Further, outcomes of patients with MPN who underwent wedge resection or segmentectomy were evaluated.

We present the following article in accordance with the STROBE reporting checklist (available at http://dx.doi. org/10.21037/jtd-20-3005).

\section{Methods}

\section{Patients selection}

This is a single-center retrospective study of the outcomes of early-stage adenocarcinoma patients after wedge resection. We prospectively collected patient data and retrospectively observed the patients. Data between October 2014 and September 2015 were retrieved from the Department of Thoracic Surgery, National Cancer Center database. The study was conducted in accordance with the Declaration of Helsinki (as revised in 2013). This study was approved by ethics committee of National Cancer Center/ Cancer Hospital, Chinese Academy of Medical Sciences and Peking Union Medical College (approval number: 1911371921). The requirement of patients' informed consent was waived owing to the retrospective nature of the study.

Inclusion criteria were as follows (Figure 1): (I) surgical approach was wedge resection; (II) clinical stage was T1a-bN0; (III) the pathological type was primary adenocarcinoma (for multifocal NSCLC, each resected 
lesion was pathologically confirmed as independent adenocarcinoma). Exclusion criteria were: (I) the pathological type is atypical adenomatous hyperplasia (AAH), adenocarcinoma in situ (AIS) or minimally adenocarcinoma (MIA); (II) tumor size is larger than $2 \mathrm{~cm}$; (III) insufficient margin distance: including positive surgical margin, $\mathrm{MD}$ $<20 \mathrm{~mm}$ or less than a maximum diameter of the tumor; (IV) patients with previous treatment for lung cancer.

\section{Radiological evaluation}

In solitary pulmonary nodule (SPN), tumor size was defined as the maximum diameter of the lesion on CT scan. In MPNs, tumor size was defined as the maximum diameter of the main focus on CT scan. Main focus was defined as the main lung cancer to be surgically resected from its tumor size and radiological invasiveness. The comparison of clinicopathologic characteristics between SPN and MPN patients in this cohort is shown in Table S1. We examined consolidation tumor ratio (CTR) from the lung window of thin-section CT. Radiological criteria of CTR 0.5 as a cut-off was used in this study and cases were classified into two subgroups: GGO-dominant $(\mathrm{CTR} \leq 0.5)$ and soliddominant (CTR $>0.5$ ) based on previous studies $(26,27)$.

\section{Surgical procedure}

Surgeries selected in this study were performed using thoracoscopic approach (single-port or three-port) without conversion to thoracotomy. Wedge resection or segmentectomy were performed to remove the main foci as well as secondary lesions on the same side. The indications for these patients underwent wedge resection are shown in Figure S1. And anatomic segmentectomy was performed for the segmentectomy group for main focus of MPN. Tumor characteristics and surgeon preference inform which sublobar procedure to perform (i.e., wedge resection or segmentectomy).

For wedge resection, we use preoperative CT-guided coil or intraoperative finger palpation to localize nodules. For segmentectomy, Three-dimensional reconstruction was used to ascertain the location of the nodules and the appropriate target segment. A systematic mediastinal nodal sampling was performed in all solid-dominant cases. Lymph node sampling was optional for GGO-dominant lesions.

\section{Pathologic diagnosis and postoperative follow-up}

Pathological sections used in this study were reviewed by an experienced senior pathologist. Pathologic diagnosis was based on the 2015 World Health Organization (WHO) classification of lung cancer. Staging standard was based on the 8th edition of the Union for International Cancer Control/American Joint Committee on Cancer (UICC/ AJCC) TNM staging for NSCLC $(28,29)$. Cases with MPN were clinically and pathologically diagnosed as multiple primary lung cancer according to the 8 th edition of the International Staging System of Lung Cancer as proposed by the AJCC (30). The degree of tumor histological differentiation was divided into three grades: I, welldifferentiated; II, moderately differentiated; III, poorly differentiated.

Follow-up data on all patients were obtained from latest medical records or imaging examinations. Out-patient review was conducted every 3 months for the first 2 years, every 6 months for the subsequent 2 years, and annually thereafter for a lifetime. Overall survival (OS) was calculated from surgery to death irrespective of cause of death. Lung cancer-specific survival (LCSS) was calculated from surgery to death due to lung cancer. Progression-free survival (PFS) was recorded as number of months from surgery to the time recurrence or metastasis was first confirmed by assessment of imaging examination. Censoring was calculated as the patients who were still alive or "lost to follow-up."

\section{Statistical analysis}

Pearson Chi-square test or Fisher's exact test was used to compare categorical variables. Mann-Whitney $\mathrm{U}$ test was used to compare continuous variables using SPSS 18.0 software (IBM Corporation, Armonk, NY, USA). Continuous variables were presented as median (interquartile range). Survival curves of 5-year OS, PFS, and LCSS were generated using Kaplan-Meier method and statistically analyzed using the log-rank test. Univariate and multivariate analyses using Cox proportional-hazards models were carried out to identify significant clinical predictors of outcome using R 3.6.2 (https://www.r-project.org/).

For further comparison of the effect of resection type on synchronous multifocal nodules, patients undergoing segmentectomy were extracted from our database as controls. A flow diagram of patient selection process is provided separately (see Figure S2). Propensity score matching (PSM) method was used to eliminate selection biases. In this study, the propensity score was calculated based on preoperative factors such as sex, age, tumor size (based on main focus), preoperative CT features (based on 
Table 1 Patients' clinicopathologic characteristics of wedge resection

\begin{tabular}{|c|c|}
\hline Clinicopathologic characteristic & Value \\
\hline Age, y (range) & $61[52-69]$ \\
\hline BMI, (range) & $23.9(22.1-26.3)$ \\
\hline Tumor size, cm (range) & $1.3(0.9-1.5)$ \\
\hline \multicolumn{2}{|l|}{ Gender, n (\%) } \\
\hline Female & $124(65.3)$ \\
\hline Male & $66(34.7)$ \\
\hline \multicolumn{2}{|l|}{ Smoking history, n (\%) } \\
\hline Ever & $48(25.3)$ \\
\hline Never & $142(74.7)$ \\
\hline \multicolumn{2}{|l|}{ Family history of carcinoma, $\mathrm{n}(\%)$} \\
\hline Yes & $68(53.0)$ \\
\hline No & $122(49.0)$ \\
\hline \multicolumn{2}{|l|}{ Clinical T stage, n (\%) } \\
\hline cT1a & $141(74.2)$ \\
\hline$c T 1 b$ & $49(25.7)$ \\
\hline \multicolumn{2}{|l|}{ If multiple, $\mathrm{n}(\%)$} \\
\hline Yes & $91(47.9)$ \\
\hline No & $99(52.1)$ \\
\hline \multicolumn{2}{|l|}{ Imaging feature, n (\%) } \\
\hline GGO-dominant & $133(70.0)$ \\
\hline Solid-dominant & $57(30.0)$ \\
\hline \multicolumn{2}{|l|}{ Pathologic T stage, n (\%) } \\
\hline $\mathrm{pT} 1 \mathrm{a}$ & $84(44.2)$ \\
\hline pT1b & $58(30.5)$ \\
\hline pT2a [Vis $\mathrm{Pl}]$ & $48(25.3)$ \\
\hline $\begin{array}{l}\text { Number of cases with systematic } \\
\text { mediastinal nodal sampling, } \mathrm{n}(\%)\end{array}$ & $104(54.7)$ \\
\hline LN sampling number, $\mathrm{n}$ (range) & $3.5(0-7)$ \\
\hline \multicolumn{2}{|l|}{ Pathologic N stage, n (\%) } \\
\hline No/Nx & $188(98.9)$ \\
\hline $\mathrm{N} 1 / 2$ & $2(1.1)$ \\
\hline \multicolumn{2}{|l|}{ Histological differentiation, n (\%) } \\
\hline Grade I & $116(61.1)$ \\
\hline Grade II & $54(28.4)$ \\
\hline Grade III & $20(10.5)$ \\
\hline
\end{tabular}

Table 1 (continued)
Table 1 (continued)

\begin{tabular}{lc}
\hline Clinicopathologic characteristic & Value \\
\hline Vis PI, n (\%) & $48(25.3)$ \\
Yes & $142(74.7)$ \\
No & \\
MVI, n (\%) & $7(3.7)$ \\
Yes & $183(96.3)$ \\
No & $125(65.8)$ \\
Pathological subtype, n (\%) & $47(24.7)$ \\
Lepidic/Acinar & $9(4.7)$ \\
Papillary & $9(4.7)$ \\
Micropapillary & \\
Mucinous adenocarcinoma & \\
\hline BMI, body mass index; MPN, multiple pulmonary nodules; \\
MVI, microscopic vascular invasion; OS, overall survival; PFS, \\
progression-free survival; Vis PI, visceral pleura invasion.
\end{tabular}

main focus), and clinical $\mathrm{T}$ stage, which may influence the choice for surgical modes. Patients in the wedge resection group and segmentectomy group were matched in a 1:1 ratio according to their propensity scores. $\mathrm{P}<0.05$ was considered statistically significant.

\section{Results}

\section{Clinicopathologic characteristics and oncology outcomes}

In this study, all 190 patients with LUAD who underwent wedge resection had a median postoperative follow-up period of 51 months (interquartile range 45.5-59 months). Clinicopathologic characteristics of patients are summarized in Table 1. In this study, the cut-off value of tumor size was set at $1.2 \mathrm{~cm}$ whereas sensitivity and specificity of PFS were $85 \%$ and $52.4 \%$, respectively.

The cumulative 5-year OS, PFS and LCSS were $95.5 \%$, $87.9 \%$, and $97.7 \%$, respectively. A total of eight patients died during the follow-up period. Out of the eight deaths, four were lung cancer-specific deaths whereas the other four from were due to other causes (cardiovascular or cerebrovascular accidents and postoperative respiratory failure). Twenty patients showed tumor progression during follow-up. Details on the 20 patients are shown in Table S2. 
Table 2 Univariate and multivariate analysis of PFS after wedge resection

\begin{tabular}{|c|c|c|c|c|c|c|}
\hline Variables & \multicolumn{3}{|c|}{ Univariate analysis } & \multicolumn{3}{|c|}{ Multivariate analysis } \\
\hline Gender (reference: female) & 2 & 0.13 & $0.82,4.7$ & & & \\
\hline Age & 1 & 0.59 & $0.97,1.1$ & & & \\
\hline Smoking history (reference: never) & 2.1 & 0.09 & $0.88,5.3$ & & & \\
\hline BMI & 0.93 & 0.31 & $0.82,1.1$ & & & \\
\hline Vis PI (reference: no) & 3.8 & $<0.01$ & $1.6,9.1$ & 1.5 & 0.42 & $0.56,4.02$ \\
\hline MVI (reference: no) & 5 & 0.01 & $1.4,17$ & 0.57 & 0.45 & $0.14,2.41$ \\
\hline \multicolumn{7}{|l|}{ If multiple (reference: SPN) } \\
\hline$>1.2 \mathrm{~cm}$ & 3 & 0.03 & $1.1,7.7$ & 2.06 & 0.16 & $0.76,5.61$ \\
\hline \multicolumn{7}{|c|}{ Histological differentiation (reference: grade I) } \\
\hline Grade II & 6 & $<0.01$ & $1.86,25.41$ & 0.9 & 0.91 & $0.16,4.98$ \\
\hline Grade III & 18 & $<0.01$ & $4.94,70.4$ & 1.9 & 0.48 & $0.31,11.74$ \\
\hline \multicolumn{7}{|c|}{ Imaging features (reference: GGO-dominant) } \\
\hline Solid-dominant & 26 & $<0.01$ & $5.9,110$ & 15.87 & 0.004 & $2.38,105.84$ \\
\hline \multicolumn{7}{|c|}{ Pathological subtype (reference: lepidic/acinar) } \\
\hline Papillary & 1.47 & 0.46 & $0.53,4.05$ & & & \\
\hline
\end{tabular}

BMI, body mass index; HR, hazard ratio; MPN, multiple primary nodules; MVI, microscopic vascular invasion; SPN, solitary pulmonary nodule; Vis PI, visceral pleura invasion.

\section{Independent prognostic factors of patients}

Cox proportional hazards model was used to analyze independent prognostic factors of PFS. Analysis of OS and LCSS was not included due to good outcomes of this well-selected cohort. This oncological outcome showed that wedge resection has a satisfactory 5 -year survival for appropriately selected patients with early-stage LUAD.

Results from univariate analysis showed that MPNs, visceral pleura invasion (Vis PI), microscopic vascular invasion $(\mathrm{MVI})$, tumor size $(>1.2 \mathrm{~cm})$, histological differentiation, and imaging features (solid-dominant) had significant effects on PFS (Table 2). Notably, MPN (vs. SPN) were associated with poor PFS (80.9\% vs. $94.1 \%$, $\mathrm{P}<0.01)$. Parameters obtained from histopathology, including Vis PI $(\mathrm{P}=0.003)$ and MVI $(\mathrm{P}=0.01)$, were significant, however pathological subtype (all $\mathrm{P}>0.05$ ) was not significant. Analysis on factors associated with imaging features, showed that patients with GGO-dominant lesions had superior PFS compared with patients with soliddominant lesions $(98.3 \%$ vs. $63.5 \%, \mathrm{P}<0.01)$. Moreover, analysis of tumor histological differentiation showed that moderately differentiated $(\mathrm{P}<0.01)$ and poorly differentiated $(\mathrm{P}<0.01)$ adenocarcinoma had lower PFS compared with well-differentiated adenocarcinoma. Tumor size larger than $1.2 \mathrm{~cm}$ had significant effect on PFS ( $\mathrm{P}=0.03)$.

Multivariable analysis results based on the Cox model are shown in Table 2. MPN [hazard ratio (HR) 3.07; 95\% confidence interval (95\% CI), 1.05-8.98; $\mathrm{P}=0.04]$ and solid-dominant lesions (HR 15.87; 95\% CI, 2.38-105.84; 

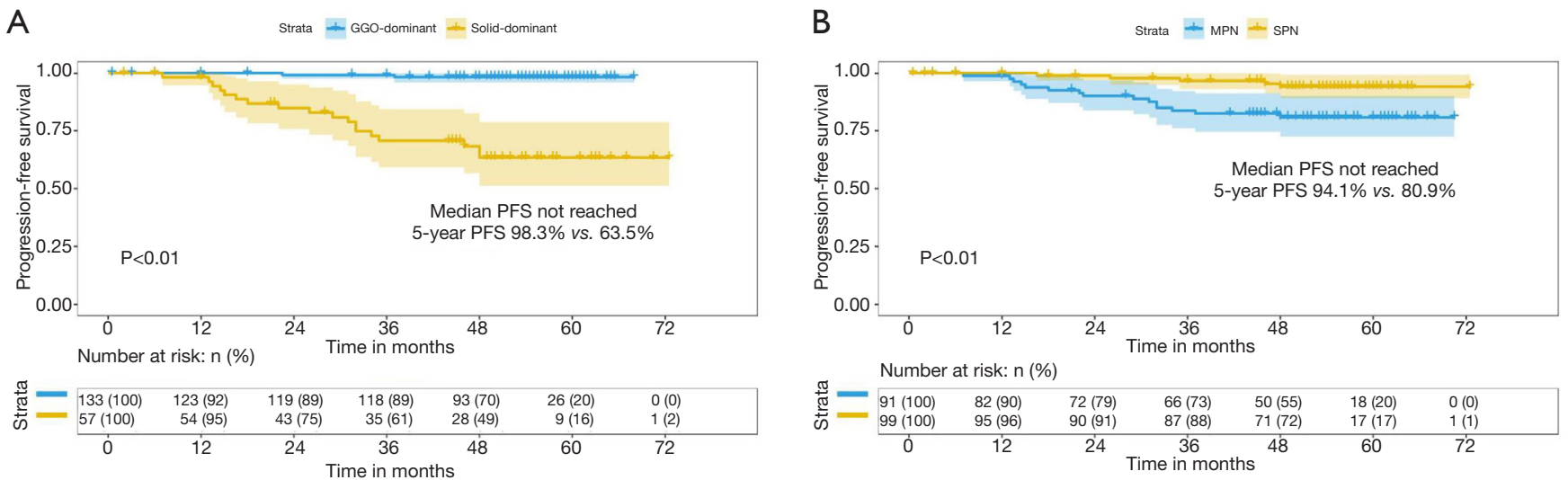

Figure 2 Progression-free survival of 190 patients underwent wedge resection. (A) Patients in GGO-dominant group had superior PFS compared with patients in solid-dominant group (98.3\% vs. $63.5 \%, \mathrm{P}<0.01)$. (B) Patients in MPN group had worse PFS compared with patients in SPN group (80.9\% vs. 94.1\%, $\mathrm{P}<0.01)$. MPN, multiple pulmonary nodule; SPN, solitary pulmonary nodules; PFS, progressionfree survival.

$\mathrm{P}=0.004)$ were the only independent prognostic factors associated with poor PFS (Figure 2).

\section{Analysis of propensity-matched groups}

To further understand the effect of surgical type on PFS in patients with MPN, a series of patients who had undergone segmentectomy were selected from our database (see Figure S2). Baseline Characteristics of MPN before propensity score matching is showed in Table S3. We hypothesized that wedge resection and segmentectomy are associated with comparable oncologic outcomes for patients with multiple primary lung cancer. A propensity-matched analysis was performed between wedge resection group (91 cases) and segmentectomy group (125 cases). After matching, clinicopathologic characteristics of the matched patients (91 cases in each group) were evaluated as shown in Table 3.

Patients with MPN undergoing segmentectomy were more likely to perform systematic mediastinal nodal sampling $(95.6 \%$ vs. $59.3 \%, \mathrm{P}<0.001)$ and had more totally LNs sampled compared with wedge resection group (10 vs. $4, \mathrm{P}<0.001)$. Therefore, patients in wedge resection group had a marginally lower frequency of $\mathrm{pN} 1 / \mathrm{N} 2$ compared with segmentectomy group $(1.1 \% v s .6 .6 \%, \mathrm{P}=0.054)$. At a median follow-up of 51 months, tumor progression was noted in five patients undergoing segmentectomy and fifteen patients undergoing wedge resection. Patients with MPN in segmentectomy group had better PFS than wedge resection group (94\% vs. 80.9\%, $\mathrm{P}=0.008$ ) (Figure 3).

\section{Discussion}

In this retrospective study, patients with early-stage adenocarcinoma who were well-selected could have an excellent 5-year OS and PFS after wedge resection. CTR $>0.5$ and MPNs were identified as two independent factors for PFS for wedge resection cohort. Therefore, wedge resection may be more suitable to patients with solitary nodules $\leq 2 \mathrm{~cm}$ with a CTR $\leq 0.5$. In addition, the 5 -year PFS of segmentectomy group were significantly higher than wedge resection group in patients with MPN. These findings imply that precise preoperative planning should be done before carrying out resections of MPNs.

Several studies on prognosis after wedge resection in patients with early stage lung cancer have been carried out. The outcomes of 5-year OS and LCSS in our study were comparable to or better than most previous studies $(14,20,21,31)$. In 2017, JCOG0804 study reported its primary endpoint that sublobar resection, mainly wedge resection, offered sufficient local control and RFS (99.7\%) for peripheral NSCLC $(\leq 2 \mathrm{~cm}, \mathrm{CTR} \leq 0.25)$ on TSCT. In this study, GGO-dominant SPN results $(\leq 2 \mathrm{~cm}$, CTR $\leq 0.5$, 5-year RFS 98.3\%) agreed with JCOG0804 survival outcomes results. However, findings from previous studies on wedge resection only apply to cohorts of patients with solitary nodule. Patients in our cohort were consciously selected for wedge resection, and it may be clinically significant given the increase in the proportion of multifocal nodules. 
Table 3 Characteristics of propensity-matched groups

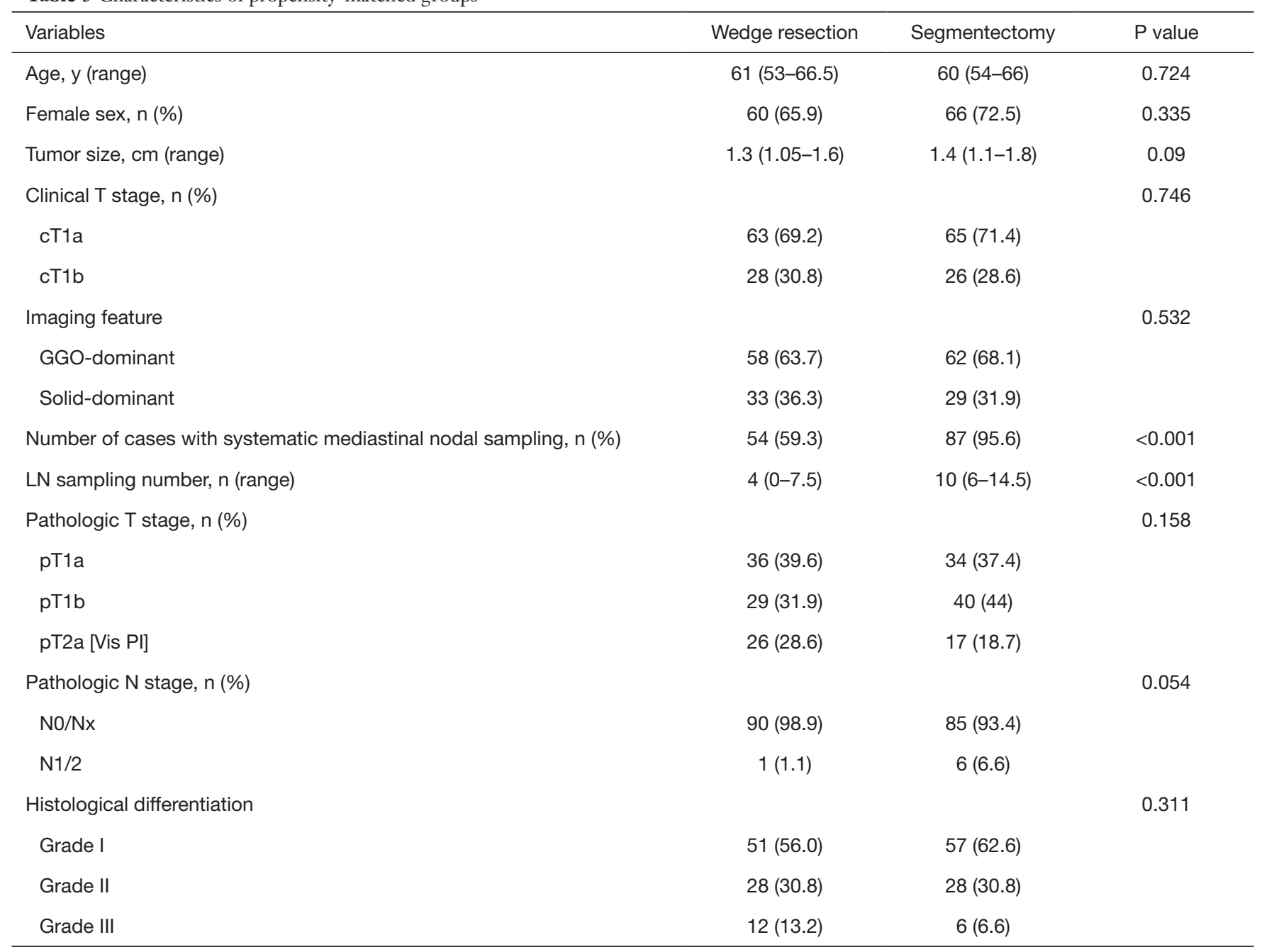

LN, lymph node.

In the current cohort, CTR, which can be considered an index of invasive component, was selected as one of prognostic factors by Cox proportional-hazards model. The correlation between CTR and their prognosis has been widely studied. Most previous studies reported that CTR is a significant prognostic factor in early NSCLC. Tsunezuka $e t a l$. reported that patients undergoing wedge resection with solid tumor size larger than $1.2 \mathrm{~cm}$ have a risk of recurrence (9). Tsutani et al. (21) reports that wedge resection has comparable surgical outcomes with segmentectomy for peripheral pulmonary nodules with CTR $\leq 0.5$. However, Cho et al. (32) suggested that wedge resection should be cautiously performed for nodules with CTR $>0.25$, due to higher recurrence rate compared that nodules with CTR $\leq 0.25$ (15.3\% vs. $1.4 \%)$. Given that lack of resection margin status and small sample size, the data from Choi's report have to be reconsidered with some skepticism. In this context, we excluded insufficient-margin cases and relatively large sample size were used over a short period of time to ensure homogeneity in treatment paradigms, staging, and practices.

Another prognostic factor of PFS in our cohort was MPNs, which has not been fully explored in previous studies. Yu et al. (33) compared the outcomes of patients with synchronous multiple primary lung cancers and solitary primary lung cancer (SMPLC). They found that tumor size was the only independent prognostic factor for SMPLC after surgical intervention. However, these findings are not comparable to the finding of the present study as the tumor size (tumors size $\geq 3 \mathrm{~cm}$ accounted for more than 


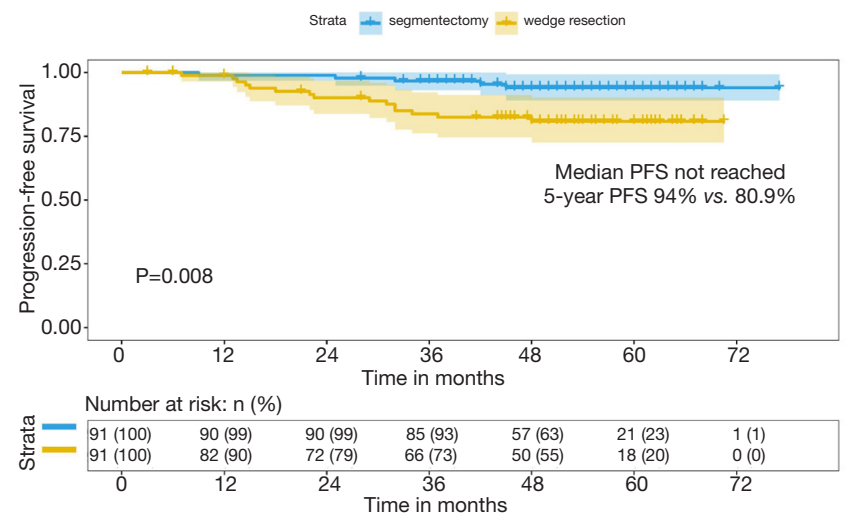

Figure 3 Progression-free survival of MPN patients underwent wedge resection and segmentectomy. Patients with MPN in segmentectomy group had better PFS than wedge resection group (94\% vs. 80.9\%, $\mathrm{P}=0.008$ ). PFS, progression-free survival.

$70 \%)$ was significantly larger than the tumor size of our cohort and the rate of sublobar resection was only $14.4 \%$. The reason why MPNs had a worse PFS in our study may as follows: First, patients with MPNs had a longer period of follow-up than SPN, which may contribute to growth of main lesion and thus increase the invasive component. Second, diagnosis of multiple primary LUAD in the current cohort was mainly based on preoperative imaging features and pathological evaluation. Genomic analysis was not used for all multifocal nodules to differentiate metastases from multiple primaries. MPNs with solid component may metastasize through aerogenous spread (34), also referred as tumor spread through air spaces (STAS), which may affect tumor staging and management. Finally, since we performed synchronous multiple pulmonary wedge resections for MPNs and observed that the extent of main focus resection may be affected by other lesions. Therefore, wedge resection may not be effective and segmentectomy or lobectomy procedures may be more effective for MPN patients.

Our preliminary findings suggested that poor PFS of MPNs may be caused by surgical mode, therefore, we further compared wedge resection and segmentectomy procedures in patients with MPNs. Theoretically, patients with the same propensity score should have the same possibility of receiving wedge resection or segmentectomy. Therefore, results obtained using PSM method may increase the credibility of the conclusions and provide useful guidance (35). After PSM, Kaplan-Meier analysis showed that PFS of segmentectomy is significantly higher compared with that of wedge resection in patients with MPNs. Several studies reported that segmentectomy should be the preferred technique for limited resection of patients with early-stage NSCLC $(36,37)$. However, these studies did not take account of MPNs. In the current study, systematic mediastinal nodal sampling was performed in $95.6 \%$ of patients who underwent segmentectomy compared to only $59.3 \%$ of patients who had a wedge resection. This ratio is consistent with reports by Altorki et al. and the American College of Surgical Oncology Group (ACOSOG) Z4032 trial (20,38). Interestingly, both studies on small solitary tumor did not translate more lymph node assessment into more nodal upstaging or an improvement in OS or PFS. A reasonable explanation maybe that main focus in MPNs have a higher malignant potential compared with solitary lung tumor due to a longer follow-up period. Therefore, lower rate of nodal sampling may lead to occult metastases neglected, which would be reflected in lower rate of adjuvant chemotherapy received after surgery. In addition, segmentectomy were always carefully designed through three-dimensional reconstruction before surgeries. This would result to a better parenchymal resection margin compared to wedge resection. Therefore, more reasonable preoperational planning for limited resection in multifocal pulmonary adenocarcinoma should be emphasized and segmentectomy procedure is recommended.

The limitation of this study is its retrospective nature. First, as this study was designed as a single-center trial, it might not have the generalizability a multi-center trial typically retains. Second, the location of the main lesion and the number of nodules was not considered as a variable in patients with MPNs which might affect invasiveness and metastasis of lymph nodes and influence effectiveness of lymph node resection (39). Finally, the follow-up period for the cohort should be extended to obtain enough survival data. Further, randomized controlled trials should be carried out to evaluate the outcomes of MPN.

\section{Conclusions}

In summary, wedge resection is a practical option for appropriately selected early-stage adenocarcinoma, with tumor size less than $2 \mathrm{~cm}$ and a CTR $\leq 0.5$. Multivariate analysis demonstrated that CTR and MPN are two independent prognostic factors affecting the surgical outcome of wedge resection. Therefore, for MPN which is not suitable for lobectomy, segmentectomy should be the surgical procedure of first choice. Regarding the lack of OS 
in this study, we will continue to follow-up these patients for more than ten years to obtain conclusive results. In addition, further studies should be carried out to explore the appropriate surgical procedure for multifocal nodules.

\section{Acknowledgments}

Funding: This work was supported by National Key R\&D Program of China (2017YFC1308700), Institutional Fundamental Research Funds (2018PT32033) and ETHICON.Excellent in surgery grant (2018-011-ZZ).

\section{Footnote}

Reporting Checklist: The authors have completed the STROBE reporting checklist. Available at http://dx.doi. org/10.21037/jtd-20-3005

Data Sharing Statement: Available at http://dx.doi. org/10.21037/jtd-20-3005

Conflicts of Interest: All authors have completed the ICMJE uniform disclosure form (available at http://dx.doi. org/10.21037/jtd-20-3005). The authors have no conflicts of interest to declare.

Etbical Statement: The authors are accountable for all aspects of the work in ensuring that questions related to the accuracy or integrity of any part of the work are appropriately investigated and resolved. The study was conducted in accordance with the Declaration of Helsinki (as revised in 2013). This study was approved by ethics committee of National Cancer Center/Cancer Hospital, Chinese Academy of Medical Sciences and Peking Union Medical College (approval number: 191137-1921). The requirement of patients' informed consent was waived owing to the retrospective nature of the study.

Open Access Statement: This is an Open Access article distributed in accordance with the Creative Commons Attribution-NonCommercial-NoDerivs 4.0 International License (CC BY-NC-ND 4.0), which permits the noncommercial replication and distribution of the article with the strict proviso that no changes or edits are made and the original work is properly cited (including links to both the formal publication through the relevant DOI and the license). See: https://creativecommons.org/licenses/by-nc-nd/4.0/.

\section{References}

1. Bray F, Ferlay J, Soerjomataram I, et al. Global cancer statistics 2018: GLOBOCAN estimates of incidence and mortality worldwide for 36 cancers in 185 countries. CA Cancer J Clin 2018;68:394-424.

2. Siegel RL, Miller KD, Jemal A. Cancer statistics, 2020. CA Cancer J Clin 2020;70:7-30.

3. Cahan WG. Radical lobectomy. J Thorac Cardiovasc Surg 1960;39:555-72.

4. Ginsberg RJ, Rubinstein LV. Randomized trial of lobectomy versus limited resection for T1 N0 non-small cell lung cancer. Lung Cancer Study Group. Ann Thorac Surg 1995;60:615-22; discussion 622-3.

5. Hattori A, Suzuki K, Takamochi K, et al. Prognostic impact of a ground-glass opacity component in clinical stage IA non-small cell lung cancer. J Thorac Cardiovasc Surg 2021;161:1469-80.

6. Ito H, Suzuki K, Mizutani T, et al. Long-term survival outcome after lobectomy in patients with clinical T1 N0 lung cancer. J Thorac Cardiovasc Surg 2020. [Epub ahead of print]. doi: 10.1016/j.jtcvs.2019.12.072.

7. Kim YW, Lee CT. Optimal management of pulmonary ground-glass opacity nodules. Transl Lung Cancer Res 2019;8:S418-S424.

8. Moon Y, Park JK, Lee KY, et al. Prognosis after wedge resection in patients with 8 edition TNM stage IA1 and IA2 non-small cell lung cancer. J Thorac Dis 2019;11:2361-72.

9. Tsunezuka H, Kato D, Okada S, et al. Surgical outcome of wide wedge resection in poor-risk patients with clinical-N0 non-small cell lung cancer. Gen Thorac Cardiovasc Surg 2017;65:581-6.

10. Wang HH, Zhang CZ, Zhang BL, et al. Sublobar resection is associated with improved outcomes over radiotherapy in the management of high-risk elderly patients with Stage I non-small cell lung cancer: a systematic review and metaanalysis. Oncotarget 2017;8:6033-42.

11. Gould MK, Donington J, Lynch WR, et al. Evaluation of individuals with pulmonary nodules: when is it lung cancer? Diagnosis and management of lung cancer, 3rd ed: American College of Chest Physicians evidence-based clinical practice guidelines. Chest 2013;143:e93S-e120S.

12. Kent M, Landreneau R, Mandrekar S, et al. Segmentectomy versus wedge resection for non-small cell lung cancer in high-risk operable patients. Ann Thorac Surg 2013;96:1747-54; discussion 1754-5. 
13. Dziedzic R, Zurek W, Marjanski T, et al. Stage I nonsmall-cell lung cancer: long-term results of lobectomy versus sublobar resection from the Polish National Lung Cancer Registry. Eur J Cardiothorac Surg 2017;52:363-9.

14. Cao J, Yuan P, Wang Y, et al. Survival Rates After Lobectomy, Segmentectomy, and Wedge Resection for Non-Small Cell Lung Cancer. Ann Thorac Surg 2018;105:1483-91.

15. Zhang H, Liu C, Tan Z, et al. Segmentectomy Versus Wedge Resection for Stage I Non-Small Cell Lung Cancer: A Meta-analysis. J Surg Res 2019;243:371-9.

16. Ohsawa M, Tsutani Y, Fujiwara M, et al. Predicting severe postoperative complication in patients with lung cancer and interstitial pneumonia. Ann Thorac Surg 2020;109:1054-60.

17. Tuminello S, Wolf A, Taioli E, et al. Comparison of Wedge Versus Lobar Resection for Stage 1 Non-Small Cell Lung Cancer: A SEER-Medicare Analysis. Ann Thorac Surg 2018;106:1260-1.

18. Sawabata N, Kawaguchi T, Yasukawa M, et al. Recording Margin Distance Is Crucial in Limited Pulmonary Resection for Lung Cancer. Ann Thorac Surg 2018;106:1592.

19. Hou B, Deng XF, Zhou D, et al. Segmentectomy versus wedge resection for the treatment of high-risk operable patients with stage I non-small cell lung cancer: a metaanalysis. Ther Adv Respir Dis 2016;10:435-43.

20. Altorki NK, Kamel MK, Narula N, et al. Anatomical Segmentectomy and Wedge Resections Are Associated with Comparable Outcomes for Patients with Small cT1N0 Non-Small Cell Lung Cancer. J Thorac Oncol 2016;11:1984-92.

21. Tsutani Y, Miyata Y, Nakayama H, et al. Appropriate sublobar resection choice for ground glass opacitydominant clinical stage IA lung adenocarcinoma: wedge resection or segmentectomy. Chest 2014;145:66-71.

22. Chen TF, Xie CY, Rao BY, et al. Surgical treatment to multiple primary lung cancer patients: a systematic review and meta-analysis. BMC Surg 2019;19:185.

23. Mohiuddin K, Haneuse S, Sofer T, et al. Relationship between margin distance and local recurrence among patients undergoing wedge resection for small $(</=2 \mathrm{~cm})$ non-small cell lung cancer. J Thorac Cardiovasc Surg 2014;147:1169-75; discussion 1175-7.

24. Sawabata N, Maeda H, Matsumura A, et al. Clinical implications of the margin cytology findings and margin/ tumor size ratio in patients who underwent pulmonary excision for peripheral non-small cell lung cancer. Surg
Today 2012;42:238-44.

25. Wolf AS, Swanson SJ, Yip R, et al. The impact of margins on outcomes after wedge resection for stage I non-small cell lung cancer. Ann Thorac Surg 2017;104:1171-8.

26. Suzuki K, Koike T, Asakawa T, et al. A prospective radiological study of thin-section computed tomography to predict pathological noninvasiveness in peripheral clinical IA lung cancer (Japan Clinical Oncology Group 0201). J Thorac Oncol 2011;6:751-6.

27. Asamura H, Hishida T, Suzuki K, et al. Radiographically determined noninvasive adenocarcinoma of the lung: survival outcomes of Japan Clinical Oncology Group 0201. J Thorac Cardiovasc Surg 2013;146:24-30.

28. Rami-Porta R, Bolejack V, Crowley J, et al. The IASLC Lung Cancer Staging Project: Proposals for the Revisions of the T Descriptors in the Forthcoming Eighth Edition of the TNM Classification for Lung Cancer. J Thorac Oncol 2015;10:990-1003.

29. Mengoli MC, Longo FR, Fraggetta F, et al. The 2015 World Health Organization Classification of lung tumors: new entities since the 2004 Classification. Pathologica 2018;110:39-67.

30. Kim SS. The IASLC lung cancer staging project proposal for the classification of lung cancers with multiple pulmonary sites of involvement: the first step toward finding optimal treatment. J Thorac Dis 2016;8:2313-4.

31. Dai C, Shen J, Ren Y, et al. Choice of Surgical Procedure for Patients With Non-Small-Cell Lung Cancer $\leq 1 \mathrm{~cm}$ or $>1$ to $2 \mathrm{~cm}$ Among Lobectomy, Segmentectomy, and Wedge Resection: A Population-Based Study. J Clin Oncol 2016;34:3175-82.

32. Cho JH, Choi YS, Kim J, et al. Long-term outcomes of wedge resection for pulmonary ground-glass opacity nodules. Ann Thorac Surg 2015;99:218-22.

33. Yu YC, Hsu PK, Yeh YC, et al. Surgical results of synchronous multiple primary lung cancers: similar to the stage-matched solitary primary lung cancers? Ann Thorac Surg 2013;96:1966-74.

34. Li R, Li X, Xue R, et al. Early metastasis detected in patients with multifocal pulmonary ground-glass opacities (GGOs). Thorax 2018;73:290-2.

35. Rosenbaum PR, Rubin DB. Reducing Bias in Observational Studies Using Subclassification on the Propensity Score. Journal of the American Statistical Association 1984;79:516-24.

36. Smith CB, Swanson SJ, Mhango G, et al. Survival after segmentectomy and wedge resection in stage I non-smallcell lung cancer. J Thorac Oncol 2013;8:73-8. 
37. Koike T, Koike T, Yoshiya K, et al. Risk factor analysis of locoregional recurrence after sublobar resection in patients with clinical stage IA non-small cell lung cancer. J Thorac Cardiovasc Surg 2013;146:372-8.

38. Fernando HC, Landreneau RJ, Mandrekar SJ, et al. Impact of brachytherapy on local recurrence rates after sublobar resection: results from ACOSOG Z4032

Cite this article as: Ji Y, Bai G, Qiu B, Zhao L, Zhou J, Xue Q, Gao S. The surgical management of early-stage lung adenocarcinoma: is wedge resection effective? J Thorac Dis 2021;13(4):2137-2147. doi: 10.21037/jtd-20-3005
(Alliance), a phase III randomized trial for high-risk operable non-small-cell lung cancer. J Clin Oncol 2014;32:2456-62.

39. Yang CJ, Brown AB, Deng JZ, et al. The oldest old: a national analysis of outcomes for patients 90 years or older with lung cancer. Ann Thorac Surg 2020;109:350-7. 


\section{Supplementary}

Table S1 Patients' clinicopathologic characteristics in SPN and MPN group

\begin{tabular}{|c|c|c|c|}
\hline Clinicopathologic characteristic & SPN & MPN & P. value \\
\hline Male & $35(35.4)$ & $31(34.1)$ & \\
\hline Female & $64(64.6)$ & $60(65.9)$ & \\
\hline Age, y (range) & $62(50.5-72)$ & $61(53-66.5)$ & 0.435 \\
\hline Smoking history, n (\%) & & & 0.997 \\
\hline No & $74(74.7)$ & $68(74.7)$ & \\
\hline Yes & $25(25.3)$ & $23(25.3)$ & \\
\hline Family history of carcinoma, n (\%) & & & 0.299 \\
\hline Tumor size (cm), n (\%) & $1.2(0.9-1.5)$ & $1.3(1.05-1.6)$ & 0.424 \\
\hline Clinical T stage, n (\%) & & & 0.944 \\
\hline cT1a & $69(69.7)$ & $63(69.2)$ & \\
\hline cT1b & $30(30.3)$ & $28(30.8)$ & \\
\hline Imaging features, n (\%) & & & 0.071 \\
\hline GGO-dominant & $75(75.8)$ & $58(63.7)$ & \\
\hline Solid-dominant & $24(24.2)$ & $33(36.3)$ & \\
\hline $\begin{array}{l}\text { Number of cases with systematic mediastinal nodal } \\
\text { sampling, } \mathrm{n}(\%)\end{array}$ & $46(46.5)$ & $54(59.3)$ & 0.076 \\
\hline pT2a [Vis PI] & $22(22.2)$ & $26(28.6)$ & \\
\hline Pathologic N stage, n (\%) & & & 0.952 \\
\hline No/Nx & $98(99.0)$ & 90 (98.9) & \\
\hline $\mathrm{N} 1 / 2$ & $1(1.0)$ & $1(1.1)$ & \\
\hline Histological differentiation, $\mathrm{n}(\%)$ & & & 0.328 \\
\hline Grade I & $65(65.7)$ & $51(56.0)$ & \\
\hline Grade II & $26(26.3)$ & $28(30.8)$ & \\
\hline Grade III & $8(8.1)$ & $12(13.2)$ & \\
\hline
\end{tabular}

BMI, body mass index; GGO, ground-glass opacity; MPN, multiple pulmonary nodule; SPN, solitary pulmonary nodule; Vis PI, visceral pleura invasion. 


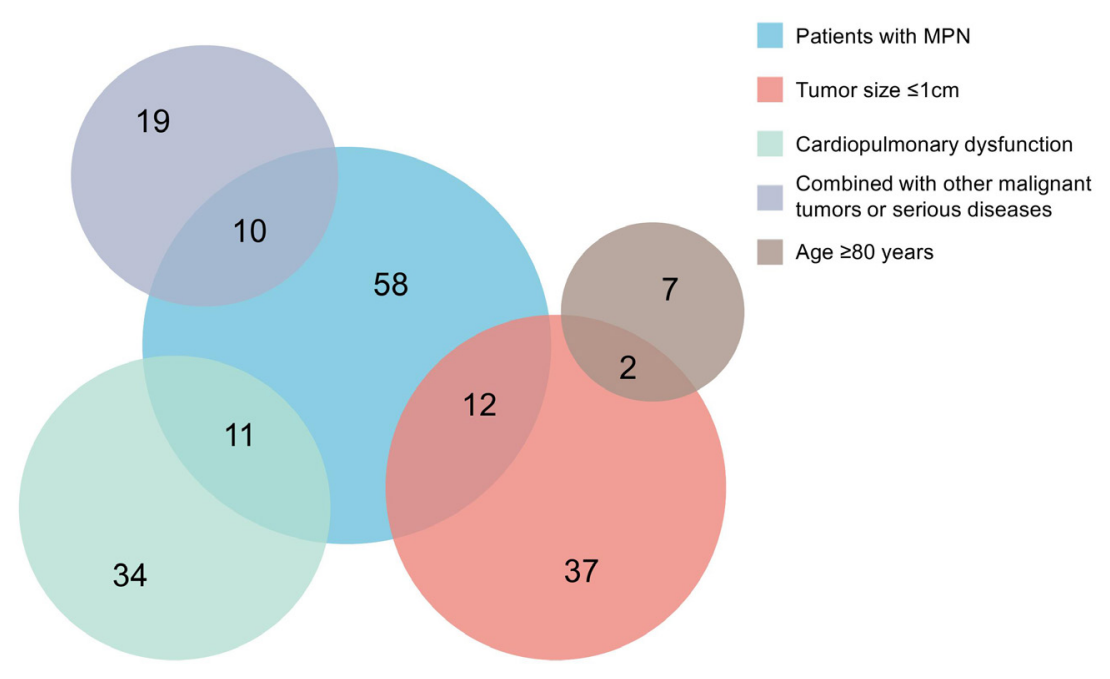

Figure S1 Venn diagram of indications for patients underwent wedge resection. MPN, multiple pulmonary nodule.

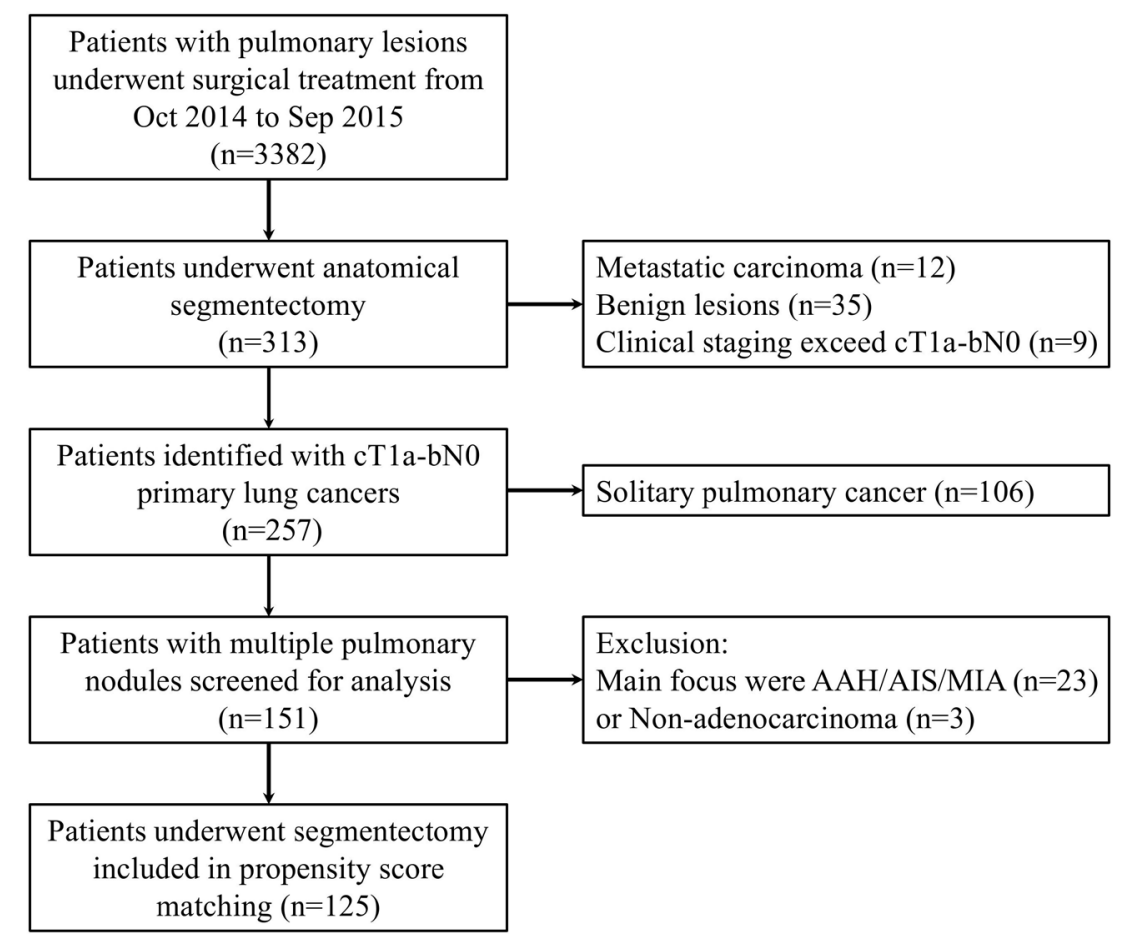

Figure S2 The flow diagram of the patient selection who underwent segmentectomy. 


\begin{tabular}{|c|c|c|c|c|c|c|c|c|c|c|}
\hline Patients with tumor progression & Sex & Age & MPN/SPN & Imaging feature & Locations of recurrence or metastasis & Vis $\mathrm{Pl}$ & MVI & Tumor size $(\mathrm{cm})$ & Histological differentiation & Pathological subtype \\
\hline 1 & Female & 67 & SPN & Solid-dominant & Pleural metastasis & Yes & No & 1.7 & Grade II & Mucinous adenocarcinoma \\
\hline 2 & Male & 68 & SPN & Solid-dominant & Pleural metastasis & Yes & No & 1.2 & Grade II & Papillary \\
\hline 3 & Male & 77 & SPN & Solid-dominant & Ipsilateral intrapulmonary metastasis & No & No & 1.2 & Grade III & Papillary \\
\hline 4 & Female & 64 & SPN & Solid-dominant & Pleural metastasis & Yes & No & 1.6 & Grade II & Papillary \\
\hline 5 & Male & 51 & SPN & Solid-dominant & Local recurrence & No & No & 1.4 & Grade I & Lepidic/Acinar \\
\hline 6 & Female & 71 & MPN & GGO-dominant & Local recurrence & No & No & 1.6 & Grade I & Lepidic/Acinar \\
\hline 7 & Male & 57 & MPN & Solid-dominant & Pleural metastasis & No & No & 1.7 & Grade II & Lepidic/Acinar \\
\hline 8 & Female & 69 & MPN & Solid-dominant & Brain metastasis & Yes & No & 1.3 & Grade III & Lepidic/Acinar \\
\hline 9 & Male & 61 & MPN & Solid-dominant & Local recurrence & Yes & Yes & 1.8 & Grade II & Micropapillary \\
\hline 10 & Female & 58 & MPN & Solid-dominant & Mediastinal lymph node metastasis & Yes & Yes & 1.6 & Grade II & Micropapillary \\
\hline 11 & Female & 45 & MPN & Solid-dominant & Local recurrence & No & No & 1.5 & Grade II & Lepidic/Acinar \\
\hline 12 & Female & 58 & MPN & Solid-dominant & Mediastinal lymph node metastasis & No & Yes & 0.9 & Grade III & Micropapillary \\
\hline 13 & Male & 61 & MPN & Solid-dominant & Adrenal metastases & Yes & No & 1.6 & Grade III & Lepidic/Acinar \\
\hline 14 & Male & 60 & MPN & GGO-dominant & Local recurrence & No & No & 1 & Grade I & Lepidic/Acinar \\
\hline 15 & Male & 62 & MPN & Solid-dominant & Local recurrence & No & No & 1.3 & Grade III & Lepidic/Acinar \\
\hline 16 & Female & 58 & MPN & Solid-dominant & Bone metastasis & Yes & No & 1.7 & Grade II & Lepidic/Acinar \\
\hline 17 & Female & 79 & MPN & Solid-dominant & Mediastinal lymph node metastasis & Yes & Yes & 1.8 & Grade III & Micropapillary \\
\hline 18 & Male & 82 & MPN & Solid-dominant & Ipsilateral intrapulmonary metastasis & Yes & Yes & 1.2 & Grade III & Mucinous adenocarcinoma \\
\hline 19 & Female & 52 & MPN & Solid-dominant & Mediastinal lymph node metastasis & Yes & No & 1.8 & Grade II & Lepidic/Acinar \\
\hline 20 & Male & 61 & MPN & Solid-dominant & Pleural metastasis & Yes & No & 1.5 & Grade II & Papillary \\
\hline
\end{tabular}

MPN, multiple pulmonary nodule; MVI, microscopic vascular invasion; SPN, solitary pulmonary nodule; Vis PI, visceral pleura invasion. 
Table S3 Baseline characteristics: wedge resection versus segmentectomy before propensity score matching

\begin{tabular}{|c|c|c|c|}
\hline Variables & Wedge resection & Segmentectomy & $P$ value \\
\hline Female sex, n (\%) & $60(65.9)$ & $105(74.5)$ & 0.161 \\
\hline Tumor size, cm (range) & $1.3(1.05-1.6)$ & $1.5(1.2-1.9)$ & 0.014 \\
\hline Clinical T stage, n (\%) & & & 0.044 \\
\hline $\mathrm{cT} 1 \mathrm{~b}$ & $28(30.8)$ & $62(44.0)$ & \\
\hline Imaging feature & & & 0.139 \\
\hline GGO-dominant & $58(63.7)$ & $76(53.9)$ & \\
\hline Solid-dominant & $33(36.3)$ & $65(46.1)$ & \\
\hline Pathologic T stage, n (\%) & & & 0.025 \\
\hline pT1a & $36(39.6)$ & $57(40.4)$ & \\
\hline $\mathrm{pT} 1 \mathrm{~b}$ & 29 (31.9) & $63(44.7)$ & \\
\hline pT2a [Vis PI] & $26(28.6)$ & $21(14.9)$ & \\
\hline Pathologic N stage, n (\%) & & & 0.053 \\
\hline No/Nx & $90(98.9)$ & $132(93.6)$ & \\
\hline $\mathrm{N} 1 / 2$ & $1(1.1)$ & $9(6.4)$ & \\
\hline
\end{tabular}

LN, lymph node. 